\section{Two edges of sickle cell disease}

\section{By Michael J. Haas, Senior Writer}

A U.S.-China team has shown that inhibiting signaling between adenosine and the adenosine $\mathrm{A}_{2 \mathrm{~B}}$ receptor in blood could prevent vasoocclusion and consequent organ damage in sickle cell disease. ${ }^{1}$ The researchers already are in talks with companies to run clinical trials of antagonists of the receptor.

Sickle cell disease results from a mutant form of $\beta$-hemoglobin (HBB) that is prone to polymerization under hypoxic conditions, resulting in the collapse of red blood cells (RBCs) into a characteristic sickle shape. The sickled RBCs can block blood vessels to cause acute episodes (vaso-occlusive crises) of pain, ischemia, tissue necrosis, organ damage and mortality.

Hydroxyurea is the only approved drug to prevent vaso-occlusive crises. However, the generic chemotherapeutic increases the risks of anemia, life-threatening infections, teratogenicity and cancer.

There are no therapies approved to treat vasoocclusive crises once they begin, and the precise molecular mechanisms underlying RBC sickling and vaso-occlusion are poorly understood.

To better understand those mechanisms and identify new disease targets, the U.S.-China team looked for molecules in blood that might drive RBC sickling.

First, metabolomic screens showed that adenosine levels were higher in the blood of sickle cell disease patients and mouse models of the disease than in blood from healthy controls.

Next, the team sought to identify which of the four known adenosine receptors interacted with adenosine to promote $\mathrm{RBC}$ sickling. They examined RBCs from transgenic mice lacking each receptor in turn to determine that adenosine $\mathrm{A}_{2 \mathrm{~B}}$ receptor ( Adora $_{2 \mathrm{~B}}$ ) — but none of the other three receptors-was involved in $\mathrm{RBC}$ sickling.

To confirm the findings in humans, the team showed that a research-grade $\mathrm{ADORA}_{2 \mathrm{~B}}$ antagonist, $\mathrm{PAB} 1115$, decreased hypoxiainduced sickling in RBCs from sickle cell patients compared with no treatment. A pegylated formulation of adenosine deaminase (PEG-ADA), which is an enzyme that metabolizes adenosine, generated similar results.

In mouse models, PAB1115 or PEG-ADA also decreased RBC sickling compared with no treatment. Treated animals experienced less vaso-occlusion and less damage to the lungs, liver, spleen and kidneys than untreated controls.

Data were reported in Nature Medicine. ${ }^{1}$

The team was led by Yang Xia, associate professor of biochemistry and molecular biology at The University of Texas Health Science Center at Houston. The group also included researchers from Central South University, The First Xiangya Hospital, The Third Xiangya Hospital, the University of Colorado Denver and Metabolon Inc., which performed the metabolomic screens in mouse blood.

Xia told SciBX her team is negotiating with undisclosed drug companies to conduct clinical trials of $\mathrm{ADORA}_{2 \mathrm{~B}}$ antagonists to prevent vaso-occlusive crises in sickle cell disease.

\section{ADORAble}

"To have a clinically meaningful impact on sickle cell disease, we believe that a good first step is the development of a drug to treat acute vasoocclusive crisis," with development of therapies to prevent crisis or treat early stage crisis as longer-term goals, said Rachel King, CEO of GlycoMimetics Inc.

John Magnani, the biotech's CSO and VP, described how an ADORA $_{2 \mathrm{~B}}$ antagonist might fit into a sickle cell disease strategy.

"An ADORA $_{2 \mathrm{~B}}$ antagonist could prevent sickling and the inflammation that results from it, but once inflammation is full-blown during vaso-occlusive crisis, preventing sickling won't be effective," he said. "Our selectin antagonist, GMI-1070, goes after a different hypoxia-induced mechanism than an ADORA $_{2 B}$ antagonist would. This suggests that the two therapeutic strategies would be compatible" for the treatment and prevention of vaso-occlusive crises, respectively.

GMI-1070 is an injectable glycomimetic inhibitor of E selectin (SELE; CD62E), P selectin (SELP; CD62P) and L selectin (SELL; CD62L) that prevents adhesion between RBCs and circulating monocytes and neutrophils. It is in Phase II testing to treat vaso-occlusive crises in sickle cell disease. The company expects the trial to finish enrolling patients this year.

GlycoMimetics also is exploring second-generation selectin antagonists for chronic use to prevent vaso-occlusive crises.

Joel Linden, a member of the Division in Inflammation Biology at the La Jolla Institute for Allergy \& Immunology (LIAI), noted that ADORA $_{2 \mathrm{~B}}$ antagonists would likely have favorable toxicity profiles. "Caffeine is a nonselective antagonist of adenosine receptors that is very widely used with only minimal side effects," such as insomnia, he said.

In August 2010, Linden and a researcher at the University of Virginia School of Medicine published in Blood that ADORA $_{2 \mathrm{~A}}$ agonists reduced infiltration of proinflammatory NK and induced NK T (iNKT) cells into the lungs of mouse models of acute chest syndrome, 
Figure 1. Adenosine's sickle cell cycle. Targeting signaling between adenosine and its receptors could help decrease disease severity and mortality in sickle cell disease.

According to a Nature Medicine study, signaling between adenosine [a] in blood plasma and the adenosine $A_{2 B}$ receptor $\left(\mathrm{ADORA}_{2 \mathrm{~B}}\right.$ ) on red blood cells (RBCs) [b] induces sickling of RBCs [c], which leads to hemolysis [d(1)] and/or vaso-occlusion [d(2)]. This results in ischemia/reperfusion, inflammation [e] and consequent damage to lungs and other organs [f].

According to a Blood study, in the lungs inflammation [e] that contributes to organ damage [f] can be decreased by agonizing ADORA $_{2 \mathrm{~A}}$ on NK cells and induced NK T (iNKT) cells [g].

Additionally, both hemolysis [d(1)] and organ damage [f] result in the release of ATP [h] into the bloodstream, which metabolizes to adenosine [a] and thereby exacerbates the disease.

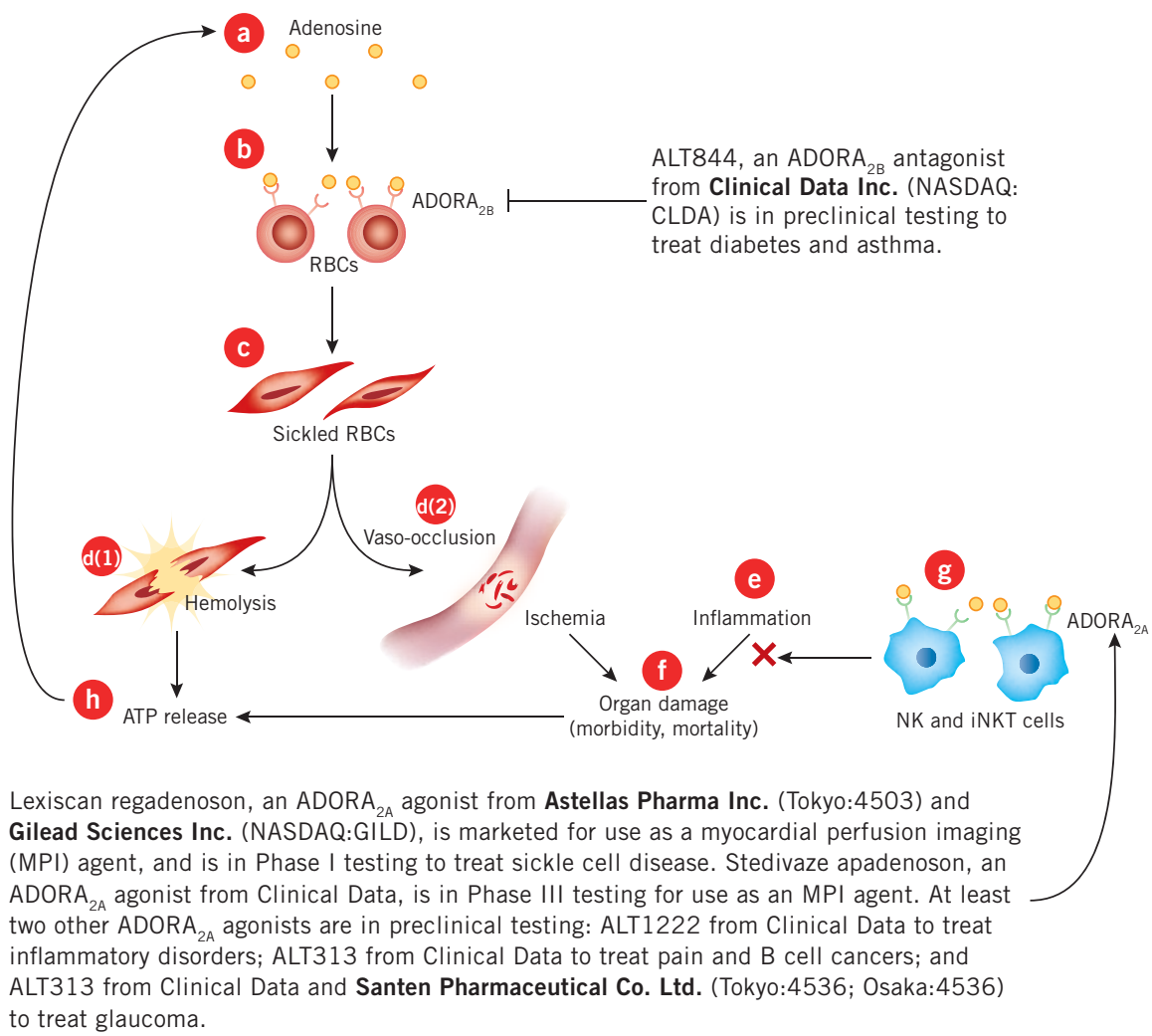

Lexiscan regadenoson, an $\mathrm{ADORA}_{2 \mathrm{~A}}$ agonist from Astellas Pharma Inc. (Tokyo:4503) and (MPI) agent, and is in Phase I testing to treat sickle cell disease. Stedivaze apadenoson, an inflammatory disorders; ALT313 from Clinical Data to treat pain and B cell cancers; and to treat glaucoma thereby improving lung function and decreasing lung damage. ${ }^{2}$ ADORA $_{2 \mathrm{~A}}$ agonists thus could help treat vaso-occlusive crisis by reducing inflammatory responses, the team wrote in its report.

Based on those data plus the new findings in Nature Medicine, Linden thinks a good approach to sickle cell disease could be "an ADORA $_{2 \mathrm{~B}}$ antagonist for long-term disease management-as a prophylactic against red blood cell sickling - and administration of an ADORA $_{2 \mathrm{~A}}$ agonist to treat" vaso-occlusive crises when they occur (see Figure 1, "Adenosine's sickle cell cycle").

Linden was a cofounder of Adenosine Therapeutics LLC, a company that developed selective adenosine receptor-targeting therapies to treat cardiovascular, inflammatory, neurological and other diseases. The company was acquired by Clinical Data Inc. in 2008. The deal included Stedivaze apadenoson, an $\mathrm{ADORA}_{2 \mathrm{~A}}$ agonist that now is in Phase III testing as a pharmaceutical stress agent for cardiovascular imaging.

Adenosine Therapeutics also provided several preclinical candidates, of which the lead ADORA ${ }_{2 \mathrm{~B}}$ antagonist is ALT844 to treat asthma and diabetes.

LIAI is collaborating with the Dana-Farber Cancer Institute and other institutions in a Phase I trial of Lexiscan regadenoson, an $\mathrm{ADORA}_{2 \mathrm{~A}}$ agonist from Gilead Sciences Inc. and Astellas Pharma Inc., to treat vaso-occlusion in sickle cell disease. According to Astellas spokesperson Jenny Keeney, the pharma provided some of the trial's funding but is otherwise not involved.
Gilead and Astellas market Lexiscan as a pharmaceutical stress agent for cardiovascular imaging.

The University of Texas Health Science Center has filed a patent application for the findings reported in Nature Medicine, and Xia said the IP is available for licensing or partnering.

Haas, M.J. SciBX 4(3); doi:10.1038/scibx.2011.62

Published online Jan. 20, 2011

REFERENCES

1. Zhang, Y. et al. Nat. Med.; published online Dec. 19, 2010; doi:10.1038/nm.2280

Contact: Yang Xia, The University of Texas Health Science Center at Houston, Houston, Texas

e-mail: yang.xia@uth.tmc.edu

2. Wallace, K.L. \& Linden, J. Blood 166, 5010-5020 (2010)

COMPANIES AND INSTITUTIONS MENTIONED

Astellas Pharma Inc. (Tokyo:4503), Tokyo, Japan

Central South University, Changsha, China

Clinical Data Inc. (NASDAQ:CLDA), Newton, Mass.

Dana-Farber Cancer Institute, Boston, Mass.

The First Xiangya Hospital, Changsha, China

Gilead Sciences Inc. (NASDAQ:GILD), Foster City, Calif.

GlycoMimetics Inc., Gaithersburg, Md.

La Jolla Institute for Allergy \& Immunology, La Jolla, Calif.

Metabolon Inc., Research Triangle Park, N.C.

The Third Xiangya Hospital, Changsha, China

University of Colorado Denver, Denver, Colo.

The University of Texas Health Science Center at Houston,

Houston, Texas

University of Virginia School of Medicine, Charlottesville, Va. 